\title{
Global homeomorphism and applications to the existence and uniqueness of solutions of some differential equations
}

\author{
Feng Yan-qing*, Wang Zhong-ying and Weng Chuan-jun
}

\section{"Correspondence: fengyq@czu.cn Department of Mathematics, Changzhou Institute of Technology, Jiangsu, 213002, P.R. China}

\begin{abstract}
In this paper, the global diffeomorphism theorem due to Zampieri was generalized. The concept of the basin of attraction is the main tool of our exposition in discussing the diffeomorphism between Banach spaces. The existence and uniqueness of a solution of the fourth order elliptic boundary value problem was proved by employing our generalized theorem. The results of this paper generalize some known theorems.

MSC: $35 J 40$

Keywords: Banach space; diffeomorphism; boundary value problem; the basin of attraction
\end{abstract}

\section{Introduction}

An important problem in nonlinear analysis is to find precise conditions for a local diffeomorphism $f$ between two Banach spaces $E, F$ to be a global one. Many authors have investigated this problem and have obtained results along this line, see [1-4]. The most famous conclusion among these may be the Hadamard-Levy theorem [2], it states that a continuously differentiable map $f$ between two Banach spaces $E$ and $F$, which is a local diffeomorphism and satisfies the condition

$$
\int_{0}^{\infty} \frac{d s}{\omega(s)}=\infty, \quad\left\|\left[f^{\prime}(x)\right]^{-1}\right\| \leq \omega(\|x\|)
$$

is a homeomorphism, where $\omega: R^{+} \rightarrow R^{+}$is a continuous increasing mapping.

In 1974, Plastock [3] reduced the global homeomorphism problem to one of finding the conditions for a local homeomorphism to satisfy the 'line lifting property'. This property was then shown to be equivalent to a limiting condition which was designated by condition $(\mathrm{L})$.

Definition 1.1 (condition (L)) Let $D$ an open connected set of $E$, the continuous mapping $f: D \subseteq E \rightarrow F$ satisfies condition (L) if for every continuous function $q:[0,1] \rightarrow F$ with $q(0) \in f(D)$, and for every $x_{0} \in f^{-1}(0)$, there exists a continuous function $p:[0, b) \rightarrow D$ with $p(0)=x_{0}$ such that $f(p(t))=q(t), 0 \leq t<b$, then there exists a sequence $t_{n} \rightarrow b$ as 
$n \rightarrow \infty$ such that

$$
\lim _{n \rightarrow \infty} p\left(t_{n}\right)
$$

exists and is in $D$.

The powerful generalization of the Hadamard-Levy theorem is as follows.

Theorem 1.1 Let $f: E \rightarrow$ F be a local homeomorphism. Then $f$ is a global homeomorphism of $E$ onto $F$ if and only iff satisfies condition (L).

Theorem 1.1 was employed to prove the global diffeomorphism and the existence and uniqueness of solution of boundary value problems by some authors [3-6].

Let us consider about the Cauchy problem:

$$
\dot{x}(t)=G(x(t)), \quad x(0)=x_{0}
$$

with

$$
G(x):=-f^{\prime}(x)^{-1}\left(f(x)-f\left(x_{0}\right)\right),
$$

which is equivalent to

$$
f(x(t))-f\left(x_{0}\right)=e^{-t}\left(f(x)-f\left(x_{0}\right)\right) .
$$

In [7], Shen found that the condition (L) could be replaced with $x(t)$ being defined on $[0,+\infty)$. Then the set $A$ of all the $x$ according to $x(t)$ is called the attraction basin of $x_{0}$, which means $x$ will be attracted to $x_{0}$ as $t \rightarrow+\infty$. Thus, Shen presented Theorem 1.2.

Lemma 1.1 [8] Let $x_{0} \in D$. Then for any $x \in D$, the path-lifting problem

$$
\left\{\begin{array}{l}
f\left(\gamma_{x}(t)\right)=f\left(x_{0}\right)+e^{-t}\left(f(x)-f\left(x_{0}\right)\right), \quad t \in R, \\
\gamma_{x}(0)=x, \quad \gamma_{x}(t) \in D
\end{array}\right.
$$

has a unique continuous solution $t \rightarrow \gamma_{x}(t)$ defined on the maximal open interval $I_{x}=$ $\left(t_{x^{-}}, t_{x^{+}}\right),-\infty \leq t_{x^{-}}<0<t_{x^{+}} \leq+\infty$. Moreover, the set $\left\{(x, t) \in D \times R: t \in I_{x}\right\}$ is open in $D \times R$ and the mapping $(x, t) \rightarrow \gamma_{x}(t)$ is continuous.

Theorem 1.2 Let $f: E \rightarrow$ F be a local homeomorphism. Then $f$ is a global homeomorphism of $E$ onto $F$ if and only if $\gamma_{x}(t)$ is defined on $R$ for all $x \in A$, namely, $\gamma_{x}(t)$ can also be extended to $-\infty$. Here $A=\left\{x \in D: t_{x}^{+}=+\infty\right\}$ is the basin of attraction of $x_{0}$.

In 1992, using the nonnegative auxiliary scalar coercive function, that is, continuous mappings $k: E \rightarrow R^{+}$with $k(x) \rightarrow+\infty$ as $\|x\| \rightarrow+\infty$, Zampieri [9] also generalized the Hadamard-Levy theorem to show the following situation.

Theorem 1.3 [9] The mapping $f \in C^{1}(E ; F)$ is a global diffeomorphism onto $F$ if

(1) $f^{\prime}(x) \in \operatorname{Isom}(E, F) \forall x \in E$;

(2) $\sup _{\|x\| \leq r}\left\|f^{\prime}(x)^{-1}\right\|<+\infty \forall r: 0<r<+\infty$; 
(3) there exists a locally Lipschitzian coercive function $k: E \rightarrow R^{+}$which admits all $D_{V}^{+} k(x)$, and it is such that $\sup \left\{D_{V}^{+} k(x): v=f^{\prime}(x)^{-1} u, x \in E, u \in F,\|u\|=1\right\}<+\infty$.

The purpose of this paper is to generalize the above Zampieri [9] theorems by making use of Theorem 1.2 and the attraction basin. In our main Theorem 2.2, in Section 2, we replaced Zampieri's condition (3) by the conditions:

(3) there exists a coercive function $k: E \rightarrow R^{+}$which admits the directional right derivatives $D_{V}^{+} k(x)$, for arbitrary $x, x_{0} \in E, y=f(x) \in F, v=f^{\prime}(x)^{-1}\left(y-f\left(x_{0}\right)\right)$ and a continuous mapping $h: R^{+} \rightarrow R^{+}$such that $D_{V}^{+} k(x) \leq h(k(x))$ and for any $r_{0}>0$, the maximum solution of the initial value problem

$$
\left\{\begin{array}{l}
r^{\prime}=h(r), \\
r(0)=r_{0}
\end{array}\right.
$$

is defined on $[0, a)$ and there exists a sequence $t_{n} \rightarrow a$ as $n \rightarrow \infty$ such that $\lim _{n \rightarrow \infty} r\left(t_{n}\right)=$ $r^{*}$ is finite.

Thus Theorem 1.3 is a corollary of our theorem. Our proofs are different from the proofs given by Zampieri [9]. In Section 3, we will apply our theorems to the existence and uniqueness of solutions of the fourth order elliptic boundary value problem.

\section{The main theorems}

In this paper, we denote $E, F$, Banach spaces, $D$ is an open subset of $E$. The following theorem will be employed to prove our main theorems.

Theorem 2.1 [10] Let $U(t, u)$ be continuous on an open $(t, x)$-set $H$ and $u=u^{0}$ the maximal solution of $u^{\prime}(t)=U(t, u), u(0)=u_{0}$. Let $v(t)$ be a continuous function on $\left[t_{0}, t_{0}+b\right]$ satisfying the condition $v\left(t_{0}\right) \leq u_{0},(t, v(t)) \in H$, and $v(t)$ has a derivative $v^{\prime}(t)$ on $t_{0} \leq t \leq t_{0}+b$ such that

$$
v^{\prime}(t) \leq U(t, v(t)) .
$$

Then, on a common interval of existence of $u^{0}(t)$ and $v(t)$

$$
v(t) \leq u^{0}(t)
$$

Now, we will show our main theorems.

Theorem 2.2 Let $f: D \subset E \rightarrow F$ be a $C^{1}$ mapping, if

(1) $f^{\prime}(x) \in \operatorname{Isom}(E, F) \forall x \in E$;

(2) $\sup _{\|x\| \leq r}\left\|f^{\prime}(x)^{-1}\right\|<+\infty \forall r: 0<r<+\infty$;

(3) there exists a coercive function $k: E \rightarrow R^{+}$which admits the directional right derivatives $D_{V}^{+} k(x)$ for arbitrary $x, x_{0} \in E, y=f(x) \in F, v=f^{\prime}(x)^{-1}\left(y-f\left(x_{0}\right)\right)$ and a continuous mapping $h: R^{+} \rightarrow R^{+}$such that $D_{V}^{+} k(x) \leq h(k(x))$ and for any $r_{0}>0$, the maximum solution of the initial value problem

$$
\left\{\begin{array}{l}
r^{\prime}=h(r), \\
r(0)=r_{0}
\end{array}\right.
$$


is defined on $[0, a)$ and there exists a sequence $t_{n} \rightarrow$ a as $n \rightarrow \infty$ such that $\lim _{n \rightarrow \infty} r\left(t_{n}\right)=r^{*}$ is finite. Then $f$ is a global homeomorphism of D onto $F$.

Proof Since $f^{\prime}(x) \in \operatorname{Isom}(E, F) \forall x \in E, f^{\prime}(x)^{-1}$ exists for all $\forall x \in D$. Hence $f$ is a local homeomorphism of $D$. Then, in the light of Theorem 1.2, we only need show that for all $x \in A$, $\gamma_{x}(t)$ can also be extended to $-\infty$. Namely, we need to consider the problem in the opposite direction.

Consider the path-lifting problem of the mapping $f$

$$
\left\{\begin{array}{l}
f\left(\gamma_{x}(t)\right)=f\left(x_{0}\right)+e^{-t}\left(f(x)-f\left(x_{0}\right)\right) \\
\gamma_{x}(0)=x, \quad \gamma_{x}(t) \in[0, a)
\end{array}\right.
$$

It is clear that

$$
\gamma_{x}^{\prime}(t)=-\left[f^{\prime}\left(\gamma_{x}(t)\right)\right]^{-1} e^{-t}\left(f(x)-f\left(x_{0}\right)\right) .
$$

Let $v=f^{\prime}\left(\gamma_{x}(t)\right)^{-1}\left(y-f\left(x_{0}\right)\right)$. By the assumption (3), we have $D_{V}^{+} k(x) \leq h(k(x))$ and the maximum solution $r(t)$ of (2.2) is defined on [0,a) and there exists a sequence $t_{n} \rightarrow a$ as $n \rightarrow \infty$ such that $\lim _{n \rightarrow \infty} r\left(t_{n}\right)=r^{*}$ is finite. It follows that $r(t)$ is continuous on $[0, a]$ and there is a constant $M_{1}$ such that $|r(t)| \leq M_{1}, t \in[0, a]$. By Theorem 2.1, we have

$$
\left\|k\left(\gamma_{x}(t)\right)\right\| \leq|r(t)| \leq M_{1}, \quad t \in[0, a]
$$

as well as $\left\|\gamma_{x}(t)\right\| \leq M_{2}, t \in[0, a)$ for some constant $M_{2}$, since $k$ is coercive, and let

$$
M_{3}=\sup _{\|x\| \leq M_{2}}\left\|f^{\prime}(x)^{-1}\right\|<+\infty, \quad x \in D
$$

Let $t=-h, t \in(a, 0], h \in[0,-a), a<0$, for $t_{1}, t_{2} \in(a, 0]$, we have

$$
\begin{aligned}
\left\|\gamma_{x}\left(t_{1}\right)-\gamma_{x}\left(t_{2}\right)\right\| & =\left\|\gamma_{x}\left(-h_{1}\right)-\gamma_{x}\left(-h_{2}\right)\right\|=\int_{-h_{2}}^{-h_{1}}\left\|\gamma_{x}^{\prime}(s)\right\| d s \\
& =\int_{-h_{2}}^{-h_{1}}\left\|f^{\prime}\left(\gamma_{x}(s)\right)^{-1}\right\| e^{-s}\left\|f(x)-f\left(x_{0}\right)\right\| d s \\
& \leq \int_{h_{1}}^{h_{2}}\left\|f^{\prime}\left(\gamma_{x}(-u)\right)\right\| e^{u}\left\|f(x)-f\left(x_{0}\right)\right\| d u \\
& \leq M_{3} \int_{h_{1}}^{h_{2}}\left\|f(x)-f\left(x_{0}\right)\right\| e^{u} d u \\
& \leq M_{3} e^{-a}\left\|f(x)-f\left(x_{0}\right)\right\|\left|t_{1}-t_{2}\right| .
\end{aligned}
$$

So $\gamma_{x}(t)$ is Lipschitz continuous on $(a, 0], \gamma_{x}(t)$ can also be extended to $-\infty$, and Theorem 2.2 is proved.

We find Zampieri's results (Theorem 1.3) as a consequence of Theorem 2.2.

Corollary 2.1 The mapping $f \in C^{1}(E ; F)$ is a global diffeomorphism onto $F$ if

(1) $f^{\prime}(x) \in \operatorname{Isom}(E, F) \forall x \in E$;

(2) $\sup _{\|x\| \leq r}\left\|f^{\prime}(x)^{-1}\right\|<+\infty \forall r: 0<r<+\infty$; 
(3) there exists a locally Lipschitzian coercive function $k: E \rightarrow R^{+}$which admits all $D_{V}^{+} k(x)$, and it is such that $\sup \left\{D_{V}^{+} k(x): v=f^{\prime}(x)^{-1} u, x \in E, u \in F,\|u\|=1\right\}<+\infty$.

Proof Let

$$
u=\frac{y-f\left(x_{0}\right)}{\left\|y-f\left(x_{0}\right)\right\|}
$$

From condition (3) for arbitrary $x, x_{0} \in E, y \in F, v=f^{\prime}(x)^{-1}\left(y-f\left(x_{0}\right)\right)$.

Define

$$
D_{v}^{+} k(x) \leq M
$$

Let $h(t)=M$, then the condition (3) of Theorem 2.2 is satisfied, and the corollary is proved.

\section{Applications of the main theorems}

Consider the fourth order elliptic boundary value problem

$$
\left\{\begin{array}{l}
\Delta^{2} u+f(x, u)=0, \\
u \in H_{I} \text { or } H_{I I},
\end{array}\right.
$$

where $f(x, u)$ is measurable in $x$ for all $u$ and has continuous partial derivatives in $u$ for almost all $x$.

Alexiades and Elcart [11] studied the existence of solution of the problem (3.1), and here we will prove the existence and uniqueness of solution of the problem (3.1).

We assume that $G$ is a bounded domain in $R^{n}$ with piecewise smooth boundary $\partial G$ whose principal curvatures are bounded.

Let

$$
H=\left\{u \in W^{2,2} \cap W_{0}^{1,2}, \Delta^{2} u \in L^{2}(G)\right\}
$$

and note that $H$ is a Hilbert space with norm $\|\cdot\|$ given by

$$
\|u\|^{2}=\int_{G}(\Delta u)^{2}+\int_{G}\left(\Delta^{2} u\right)^{2}, \quad u \in H
$$

where $W^{2,2} \cap W_{0}^{1,2}$ (see [12, Chapter 7]) can be shown to consist of $W^{2,2}$ limits of smooth functions which vanish on the boundary.

We will first consider the differential operator $L$ defined by

$$
L u=\Delta^{2} u+a(x) u,
$$

where $a(x)$ are bounded measurable functions defined in $G$.

We shall be concerned with the sets of boundary conditions

$$
u=\frac{\partial u}{\partial n}=0, \quad \text { on } \partial G
$$


or

$$
u=\Delta u=0, \quad \text { on } \partial G
$$

and we define

$$
H_{I}=\left\{u \in H, \frac{\partial u}{\partial n}=0, x \in \partial G\right\}, \quad H_{I I}=\left\{u \in H, \Delta u \in W_{0}^{1,2}\right\} .
$$

Each of these spaces is a closed subspace of $H$ and the differential operator $L$ is a bounded linear operator from $H_{I}$ or $H_{I I}$ into $L^{2}(G)$.

Denote

$$
\Omega=\inf \frac{\int(\Delta u)^{2}}{\int u^{2}}
$$

where the infimum is taken over $H_{I}$ or $H_{I I}$ according to whether (I) or (II) are the boundary conditions in the relevant eigenvalue problem. In fact, $\Omega$ is the first eigenvalue in the clamped plate $\left(\Delta^{2} \varphi-\Omega \varphi=0\right)$ problem.

Let

$$
m_{0}=\inf _{x \in G} a(x), \quad M_{0}=\sup _{x \in G} a(x) .
$$

Lemma 3.1 If $\inf _{x \in G} a(x)>-\Omega$, then $L$ is an invertible operator of $H_{I}$ as well as of $H_{I I}$ onto $L^{2}(G)$ and

$$
\|u\| \leq C\|L u\|_{0}
$$

where $C=2+\frac{\Omega}{\varepsilon^{2}}+\frac{2 M_{0}^{2}}{\varepsilon^{2}}(\varepsilon>0)$.

Proof Since $\Omega=\inf \frac{\int(\Delta u)^{2}}{\int u^{2}}>0$ is the first eigenvalue of $\Delta^{2} \varphi-\Omega \varphi=0$ in $H_{I}\left(H_{I I}\right)$, it follows that for all $u \in H_{I}\left(H_{I I}\right)$, zero is not an eigenvalue of $\Delta^{2} \varphi-\Omega \varphi=0$, so for every $u \in H_{I}\left(H_{I I}\right)$, the operator $L u=\Delta^{2} u+a(x) u$ is an invertible operator of $H_{I}\left(H_{I I}\right)$ onto $L^{2}(G)$.

For $u \in H_{I}\left(H_{I I}\right)$, we have

$$
\int(\Delta u)^{2}+\int a u^{2}=\int u L u \leq \frac{\varepsilon}{2} \int u^{2}+\frac{1}{2 \varepsilon} \int(L u)^{2},
$$

for any $\varepsilon>0$ and $-\Omega<m_{0}$, it follows that

$$
\begin{aligned}
& \int(\Delta u)^{2}+m_{0} \int u^{2} \leq \frac{\varepsilon}{2} \int u^{2}+\frac{1}{2 \varepsilon} \int(L u)^{2}, \\
& \int(\Delta u)^{2} \leq\left(\frac{\varepsilon}{2}-m_{0}\right) \int u^{2}+\frac{1}{2 \varepsilon} \int(L u)^{2} .
\end{aligned}
$$

From (3.3), we have

$$
\int(\Delta u)^{2} \leq\left(\frac{\varepsilon}{2 \Omega}+1\right) \int(\Delta u)^{2}+\frac{1}{2 \varepsilon} \int(L u)^{2}, \quad \int(\Delta u)^{2} \leq \frac{\Omega}{\varepsilon^{2}} \int(L u)^{2}
$$


and

$$
\begin{aligned}
\int\left(\Delta^{2} u\right)^{2} & =\int(L u-a u)^{2} \\
& \leq 2 \int(L u)^{2}+2 \int(a u)^{2} \\
& \leq\left(2+\frac{2 a^{2}}{\varepsilon^{2}}\right) \int(L u)^{2} \leq\left(2+\frac{2 M_{0}^{2}}{\varepsilon^{2}}\right) \int(L u)^{2} .
\end{aligned}
$$

We obtain

$$
\begin{aligned}
\|u\|^{2} & =\int(\Delta u)^{2}+\int\left(\Delta^{2} u\right)^{2} \\
& \leq\left(2+\frac{\Omega}{\varepsilon^{2}}+\frac{2 M_{0}^{2}}{\varepsilon^{2}}\right) \int(L u)^{2} \\
& \leq C\|L u\|_{0}^{2} .
\end{aligned}
$$

Here $C=2+\frac{\Omega}{\varepsilon^{2}}+\frac{2 M_{0}^{2}}{\varepsilon^{2}}$.

Now we express (3.1) in the form

$$
F(u)=\Delta^{2} u+f(x, u)
$$

and (3.1) is equivalent to the operator equation

$$
F(u)=0, \quad u \in H_{I}\left(H_{I I}\right) .
$$

For any $u, \varphi \in H_{I}\left(H_{I I}\right)$, we have

$$
F^{\prime}(u)(\varphi)=\Delta^{2} \varphi+f_{u}^{\prime}(x, u) \varphi .
$$

Theorem 3.1 Assume that

(1) $\inf _{G \times R} f_{u}^{\prime}(x, u)>-\Omega$;

(2) there exists a coercive function $k: R^{n} \rightarrow R^{+}$which admits the directional right derivatives $D_{V}^{+} k(x)$ for arbitrary $u, u_{0} \in H_{I}\left(H_{I I}\right), y \in L^{2}(G), v=F^{\prime}(u)^{-1}\left(y-F\left(u_{0}\right)\right)$ and a continuous mapping $h: R^{+} \rightarrow R^{+}$such that $D_{V}^{+} k(u) \leq h(k(u))$ and for any $r_{0}>0$, the maximum solution of the initial value problem

$$
\left\{\begin{array}{l}
r^{\prime}=h(r) \\
r(0)=r_{0}
\end{array}\right.
$$

is defined on $[0, a)$ and there exists a sequence $\left\{t_{n}\right\}$ such that $t_{n} \rightarrow a n \rightarrow \infty$

$$
\lim _{n \rightarrow \infty} r\left(t_{n}\right)=r^{*}
$$

is finite. Then there is a unique solution of the equation $F(u)=0$ in $H_{I}\left(H_{I I}\right)$. 
Proof The condition $\inf _{G \times R} f_{u}^{\prime}(x, u)>-\Omega$ implies that, for each $u, F^{\prime}(u)$ is invertible as a linear operator from $H_{I}\left(H_{I I}\right)$ onto $L^{2}(G)$. Furthermore, an upper bound for $\left\|F^{\prime}(u)^{-1}\right\|$ is provided by (3.4) if the coefficient $a(x)$ is identified with $f_{u}^{\prime}(x, u)$. In fact, $C=O\left(M_{0}\right)$, where $C$ is the constant in (3.4), implies that

$$
\left\|\left[F^{\prime}(u)\right]^{-1}\right\| \leq \alpha \sup _{G}\left|f_{u}^{\prime}(x, u)\right|+\beta
$$

for positive constant $\alpha, \beta$.

By the above discussion and condition (2), the assumptions of Theorem 2.2 are satisfied, $F$ is a homeomorphism of $H_{I}\left(H_{I I}\right)$ onto $L^{2}(G)$, and the equation $F(u)=0$ has a unique solution.

\section{Corollary 3.1 Assume that $f$ satisfies}

(1) $\inf _{G \times R} f_{u}^{\prime}>-\Omega$;

(2) uniformly in $x,\left\|f_{u}^{\prime}\right\|=\omega(\|u\|)$, where $\omega$ is a continuous map satisfying $\int_{a}^{\infty} \frac{d t}{\omega(t)}=\infty$.

Then there is a unique solution of (3.1) in $H_{I}\left(H_{I I}\right)$.

Proof We have from Lemma 3.1 and (3.4)

$$
\left\|\left[F^{\prime}(u)\right]^{-1}\right\| \leq \alpha \sup _{G}\left|f_{u}^{\prime}(x, u(x))\right|+\beta
$$

for positive constants $\alpha, \beta$.

Denote $\omega_{1}(t)=\alpha \omega(t)+\beta$; clearly, $\int_{a}^{\infty} \frac{d t}{\omega_{1}(t)}=\infty$.

Let

$$
k(u)=\int_{a}^{\|u\|} \frac{d t}{\alpha \sup _{G}\left|f_{u}(x, u)\right|+\beta}=\int_{a}^{\|u\|} \frac{d t}{\omega_{1}(t)}
$$

then $k(u) \rightarrow+\infty$ as $\|u\| \rightarrow+\infty$.

For $v=F^{\prime}(u)^{-1}\left(y-F\left(u_{0}\right)\right), u_{0} \in H_{I}\left(H_{I I}\right), y \in L^{2}(G)$, we have

$$
\begin{aligned}
D_{v}^{+} k(u) & =\lim _{s \rightarrow 0^{+}} \frac{k(u+s v)-k(u)}{s} \\
& =\frac{1}{\left\|\alpha \sup _{G}\left|f_{u}(x, u)\right|+\beta\right\|} \lim _{s \rightarrow 0^{+}} \frac{\|u+s v\|-\|u\|}{s}=\frac{1}{\omega_{1}(t)} \lim _{s \rightarrow 0^{+}} \frac{\|u+s v\|-\|u\|}{s} \\
& \leq \frac{1}{\left\|F^{\prime}(u)\right\|}\|v\|=\left\|y-F\left(u_{0}\right)\right\|=M .
\end{aligned}
$$

Let $h(t)=M$, the initial value problem

$$
\left\{\begin{array}{l}
r^{\prime}=h(r) \\
r(0)=r_{0}
\end{array}\right.
$$

changes to

$$
\left\{\begin{array}{l}
r^{\prime}=M, \\
r(0)=r_{0}>0
\end{array}\right.
$$


the solution of equation (3.5) on $[0, b)$ is $r(t)=M t$. Hence all the conditions of Theorem 3.1 are satisfied. We get the results of this corollary.

\section{Corollary 3.2 (Alexiades and Elcrat) Assume thatf satisfies}

(1) $\inf _{G \times R} f_{u}^{\prime}>-\Omega$;

(2) $\left\|f_{u}^{\prime}\right\|=O(\|u\|)$, uniformly in $x \in G$.

Then there is a unique solution of $(3.1)$ in $H_{I}\left(H_{I I}\right)$.

Proof Condition (2) implies that $\int_{a}^{\infty} \frac{d t}{\omega(t)}=\infty$ holds, and the result of Elcart and Sigillito in [11] becomes a special case of Theorem 3.1.

\section{Competing interests}

The authors declared that they have no conflicts of interest to this work.

\section{Authors' contributions}

All authors contributed equally to the manuscript. All authors read and approved the final manuscript.

\section{Acknowledgements}

The authors are grateful to the referees for their comments and references which improve the paper. The work has been supported by the Natural Science Foundation of Jiansu (13KJD110001).

Received: 17 October 2013 Accepted: 13 January 2014 Published: 31 Jan 2014

\section{References}

1. Meyer, GH: On solving nonlinear equations with a one-parameter operator imbedding. SIAM J. Numer. Anal. 5(3), 739-752 (1968)

2. Brown, KJ, Lin, SS: Periodically perturbed conservative systems and a global inverse function theorem. Nonlinear Anal. 4(1), 193-201 (1980)

3. Plastock, R: Homeomorphism between Banach space. Trans. Am. Math. Soc. 200, 169-183 (1974)

4. Shen, ZH: On the periodic solution to the Newtonian equation of motion. Nonlinear Anal. 13(2), 145-149 (1989)

5. Shen, ZH, Wolfe, MA: On the existence of periodic solution of periodically perturbed conservative systems. J. Math. Anal. Appl. 153(1), 78-83 (1990)

6. Radulescu, M, Radulescu, S: Global inversion theorems and application to differential equation. Nonlinear Anal. 4(4), 951-965 (1980)

7. Wang, WX, Shen, ZH: The basin of attraction in Banach spaces and its applications. Acta Math. Sin. 49(2), 1013-1020 (2006)

8. Gorni, G: A criterion of invertibility in the large for local diffeomorphisms between Banach spaces. Nonlinear Anal. 21(1), 43-47 (1993)

9. Zampieri, G: Diffeomorphisms with Banach space domains. Nonlinear Anal. 19(10), 923-932 (1992)

10. Lakshmikantham, V, Leeda, S: Differential and Integral Inequalities, vol. II. Academic Press, New York (1969)

11. Alexiades, V, Elcrat, A, Shaefer, P: Existence theorems for some nonlinear fourth order elliptic boundary value problems. Nonlinear Anal., Theory Methods Appl. 4(4), 805-813 (1979)

12. Payne, LE: Isoperimetric inequalities and their application. SIAM Rev. 9(1), 453-488 (1967)

10.1186/1687-1847-2014-52

Cite this article as: Yan-qing et al.: Global homeomorphism and applications to the existence and uniqueness of solutions of some differential equations. Advances in Difference Equations 2014, 2014:52 